\title{
Evaluation de la sensibilité de trois espèces de glossines d'élevage au D.D.T et à l'Endosulfan déterminée au moyen des applicateurs capillaires de l'O. M.S.
}

\author{
par A. BREARD (*)
}

\begin{abstract}
RÉSUMÉ
La sensibilité de 3 espèces de glossines d'élevage ( $G$. tachinoides, $G . m$. morsitans, G. p. gambiensis) au D. D. T. et à l'Endosulfan, a été évaluée par applications topiques, au moyen du tube applicateur capillaire de l'O. M.S. Celui-ci, d'un maniement très simple, permet d'aboutir à des résultats comparables, qualitativement, à ceux obtenus par d'autres auteurs au moyen d'un matériel plus sophistiqué.

L'espece de glossine la plus sensible à l'Endosulfan est $G$. tachinoides; la résistance de $G . m$. morsitans est intermédia1re entre celle de $G$. tachinoides et celle de G. p. gambiensis.

Par rapport à l'Endosulfan, le D. D. T. présente une toxicité très faible puisque les D. L. 50 obtenues avec cet insecticide sont environ 20 fois inférieures.

Des 3 espèces de glossines utilisées, $G$. tachinoides est la pius sensible aux 2 insecticides. La mortalité chez $G$. m. morsitans est intermédiaire entre $G$ tachinoides et G. p. gambiensis.
\end{abstract}

Le procédé le plus efficace et le plus largement utilisé actuellement dans la lutte contre les glossines, vecteurs et hôtes intermédiaires des trypanosomoses humaines et animales en Afrique intertropicale, est la pulvérisation, à partir du sol ou par voie aérienne, sur la végétation servant de lieux de repos et de gîtes de reproduction, d'insecticides rémanents dont les plus couramment employés sont le D. D. T., la Dieldrine pour leur toxicité résiduelle, et l'Endosulfan dont l'action fugace est compensée par une toxicité immédiate prononcée.

Cependant, l'utilisation de ces produits ne peut plus être recommandée en raison des risques de pollution qu'elle implique à long terme dans le milieu et dans la chaîne alimentaire.

${ }^{*}$ ) Institut d'Elevage et de Médecine Vétérinaire des Pays Tropicaux, Laboratoire de Microbiologie, 10, rue Pierre-Curie, 94700 Maisons-Alfort.
Le nombre de travaux effectués, au cours de ces dernières années, sur l'évaluation de l'action d'insecticides de remplacement sur les glossines, tant au laboratoire que sur le terrain, est très limité, les travaux les plus récents étant ceux de HADAWAY (1972 (2), 1976 (3)) et HADAWAY et TURNER (1975 (4)).

Peu d'études ont également été effectuées sur les différences de sensibilité pouvant exister entre espèces (3) vis-à-vis d'un même produit, peutêtre parce que jusqu'à présent aucune résistance n'a été décelée chez les glossines et que les campagnes de lutte entreprises dans différentes régions d'Afrique ont généralement donné d'excellents résultats, du moins à court terme.

Devant la nécessité de remplacer les insecticides organochlorés qui subissent, dans de nombreux pays, des restrictions légales d'emploi et dont la production risque de ne pas être assurée à l'avenir par les principaux pays producteurs, les 
organisations internationales, telle l'O.M.S., incitent fortement les organismes de recherche à étudier de nouveaux insecticides et à déceler et mesurer la résistance aux pesticides pouvant apparaître chez $n$ "importe quelle espèce d'insecte.

Dans ce but, l'O. M. S. a mis au point des nécessaires d'épreuve et proposé des méthodes pour déterminer la sensibilité ou la résistance aux insecticides des principales espèces vectrices.

Jusqu'à ces dernières années, il n'existait pas de méthode normalisée applicable aux glossines. Les tests étaient effectués soit par contact de l'insecte sur des papiers imprégnés de solutions insecticides à diverses concentrations, au moyen de la trousse standard O. M.S. pour les moustiques adultes $(5,1,7,8)$, soit par applications locales à l'aide d'une microseringue $(2,3)$.

En 1970, J'O. M.S. a proposé une méthode utilisable pour les Muscidae (mouches domestiques, glossines, stomoxes, calliphores, etc.) et a présenté un nécessaire d'épreuve comprenant en particulier :

- des applicateurs capillaires, essentiellement composés d'un tube capillaire de $0,36 \mu \mathrm{l}$ avec tube porte-capillaire et tube de caoutchouc muni d'un embout ;

- des gammes d'insecticides à diverses concentrations, en solution dans du phtalate de dioctyle.

Nous avons utilisé cet appareıllage pour étudier la sensibilité relative de diverses espèces de glossines d'élevage à l'égard de l'Endosulfan et du D. D. T., ce qui nous a permis également d'en situer la valeur.

\section{MATÉRIEL ET MÉTHODES}

\section{a) Glossines}

Les insectes proviennent de l'élevage de glossines du laboratoire d'Entomologie de 1'1. E. M. V. T., à Maisons-Alfort.

Les tests ont été effectués sur des individus mâles, ténéraux, à jeun, dans les $24 \mathrm{~h}$ suivant leur éclosion, appartenant à 3 espèces différentes:

- Glossina tachinoides Westwood, 1850.

- Glossina morsitans morsitans. Westwood, 1850.

- Glossina palpalis gambiensis Vanderplank, 1949.
La sensibilité à l'Endosulfan a été testée sur les 3 espèces (G. tachinoides, G. m. morsitans et G. p. gambiensis).

Deux espèces seulement $(G$. tachinoides et G. m. morsitans) ont été utilisées pour tester leur sensibilité au D. D. T.

\section{b) Préparation et conservation des solutions insecticides}

Des tests préliminaires ont été effectués avec des insecticides fournis par l'O. M. S., en solution dans du dioctyle phtalate. Ils ont révélé une toxicité élevée de ce solvant pour les glossines, même après dilution à $1 / 2$ dans l'acétone, aussi bien chez les témoins que chez les mouches ayant reçu une application d'insecticide.

Un test effectué avec 2 lots de 20 mâles de G. tachinoides, l'un recevant de l'acétone pure, l'autre du dioctyle phtalate dilué à $1 / 2$ dans l'acétone, a en effet donné les résultats suivants (totaux cumulés à $48 \mathrm{~h}$ ) :

- acétone pure : 1 mort à 24 h (5 p. 100); 2 morts à 48 h (10 p. 100);

- D. O. P. + acétone : 6 morts à $24 \mathrm{~h}(30 \mathrm{p}$. $100) ; 17$ morts à 48 h $(85$ p. 100$)$.

Dans ces conditions, il a été décidé de n'utiliser que des insecticides de qualité technique, en poudre, uniquement dilués dans l'acétone pure. A partir d'une solution-mère à 1 p. 100 gardée au réfrigérateur, des solutions-filles ont été préparées par dilutions successives, afin d'obtenir des concentrations variant selon une progression géométrique de raison 2 .

Ces solutions-filles, conservées en flacons hermétiques, au congélateur, ont toujours été employées dans les 4 semaines suivantes.

Pour déterminer les doses à tester, on a pratiqué des épreuves préliminaires qui ont permis de situer l'ordre de grandeur de la D. L. 50 et de choisir les concentrations à appliquer. Les épreuves ont ensuite été effectuées avec 3 ou 4 concentrations se situant de part et d'autre de la D. L. 50, chaque épreuve étant répétée de 2 à 4 fois.

\section{c) Test}

Aussitôt après l'éclosion en salle d'élevage, les mouches étaient transférées dans des boîtes faites d'un cylindre de carton operculé à chaque 
extrémité par un disque de cellophane maintenu par un anneau de caoutchouc ; chaque boîte renfermait 20 individus et constituait un lot d'épreuve.

Les tests étaient exécutés dans le courant de l'après-midi du même jour. Les applications topiques ont été réalisées dans un temps aussi bref que possible, sur l'insecte anesthésié au $\mathrm{CO}_{2}$, au moyen de l'applicateur capillaire de l'O. M. S. - dont la manipulation est simple et rapide par dépôt, sur la face supérieure du thorax de chaque individu, d'une gouttelette de solution insecticide ou de solvant d'un volume moyen de $0,36 \mu l$.

A chaque série d'épreuve, un lot témoin (20 mouches), était manipulé dans les mêmes conditions que les lots d'épreuve, mais ne recevait, en application topique, que le solvant pur.

Ensuite, les mouches, remises dans les boîtes d'observation, étaient conservées pendant $48 \mathrm{~h}$, à jeun et à l'obscurité, dans une étuve réglée à $25^{\circ} \mathrm{C}$ et 80 p. 100 d'humidité relative.

La lecture des résultats a eu lieu, dans chaque cas, 24 et $48 \mathrm{~h}$ après l'expérience. Ont été comptées comme mortes, les mouches ne pouvant plus se déplacer normalement. Lorsque la mortalité des témoins dépassait 20 p. 100, le test était annulé.

\section{RÉSULTATS}

Le taux de mortalité, corrigé selon la formule d'Abbott $(*)$ lorsque la mortalité des témoins était comprise entre 5 et 20 p. 100, a été calculé, pour chacune des concentrations testées, à partir du nombre total d'insectes soumis aux différentes épreuves et des nombres correspondants de morts dans les $24 \mathrm{~h}$ et dans les $48 \mathrm{~h}$.

Les mortalités corrigées, correspondant aux diverses concentrations testées, ont été reportées sur papier gausso-logarithmique, de façon à obtenir une droite de HENRY (graphiques 1 à 4), à partir de laquelle on a estimé la valeur de la D. L. 50 , à 24 et à 48 h (tabl. 1 ).

(*) Mortalité des spécimens d'épreuve (en \%) - mortalité des témoins (en \%) $\times 100$ 100 - mortalités des témoins (en \%)

TABLEAU N $\mathrm{N}^{\circ} \mathrm{I}$ - D.L. 50 obtenues à $24 \mathrm{~h}$ et à $48 \mathrm{~h}$ avec le D.D.T. et l'Endosulfan.

\begin{tabular}{|c|c|c|c|c|}
\hline \multirow{3}{*}{ D.D.T. } & & G. tachinotítes & G. m. morsitans & G. p. gambiensis \\
\hline & $\begin{array}{c}\text { D.L. } 50 \\
24 \mathrm{~h} \\
\mathrm{Ng} / \text { mouche }\end{array}$ & 9,8 & 27 & - \\
\hline & $\begin{array}{c}\text { D.L. } 50 \\
48 \mathrm{~h} \\
\mathrm{Ng} / \text { mouche }\end{array}$ & 8,1 & 23,7 & - \\
\hline \multirow{2}{*}{ Endosulfan } & $\begin{array}{c}\text { D.L. } 50 \\
24 \mathrm{~h} \\
\mathrm{Ng} / \text { mouche }\end{array}$ & 0,48 & 2,1 & 3,7 \\
\hline & $\begin{array}{c}\text { D.L, } 50 \\
4 B \text { h } \\
\text { Ng/mouche }\end{array}$ & 0,31 & 1,35 & 2,6 \\
\hline
\end{tabular}

Il ressort à l'évidence des résultats obtenus, qu'il existe une différence de sensibilité importante aux 2 insecticides testés, entre espèces.

G. tachinoides est l'espèce la plus sensible.

G. m. morsitans est environ 2 fois plus sensible à l'Endosulfan que G. p. gambiensis, alors que
G. tachinoides est au moins 4 fois plus sensible à cet insecticide que ne l'est $G . m$. morsitans.

De même, $G$. tachinoides est plus sensible au D. D. T. que G. m. morsitans.

Enfin, l'Endosulfan est nettement plus toxique pour les glossines que le D. D. T. (25 fois pour G. tachinoides; 19 fois pour G. m. morsitans). 


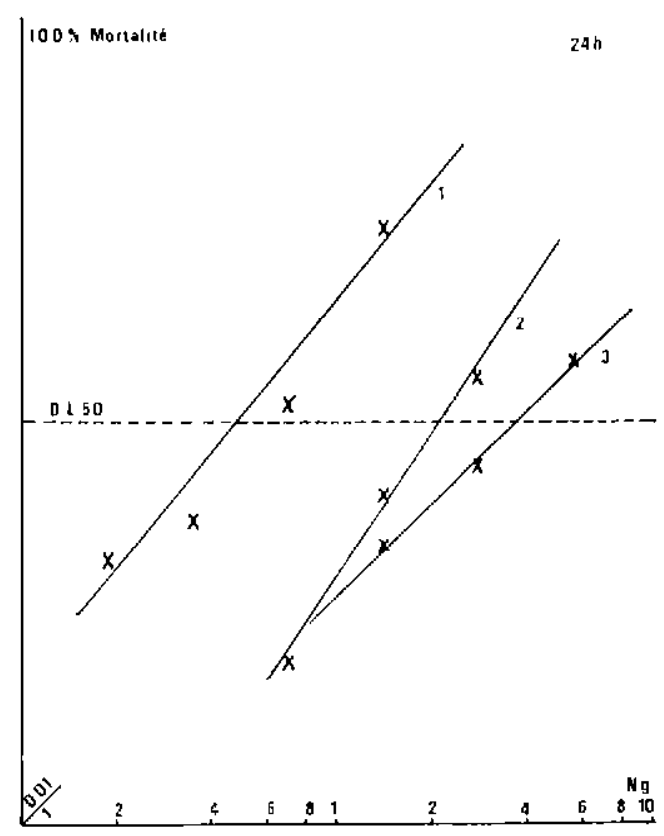

Graphique 1. - Action de l'Endosulfan sur G, tachinoides (1), G. m. morsitans (2) et G. p. gambiensis (3).

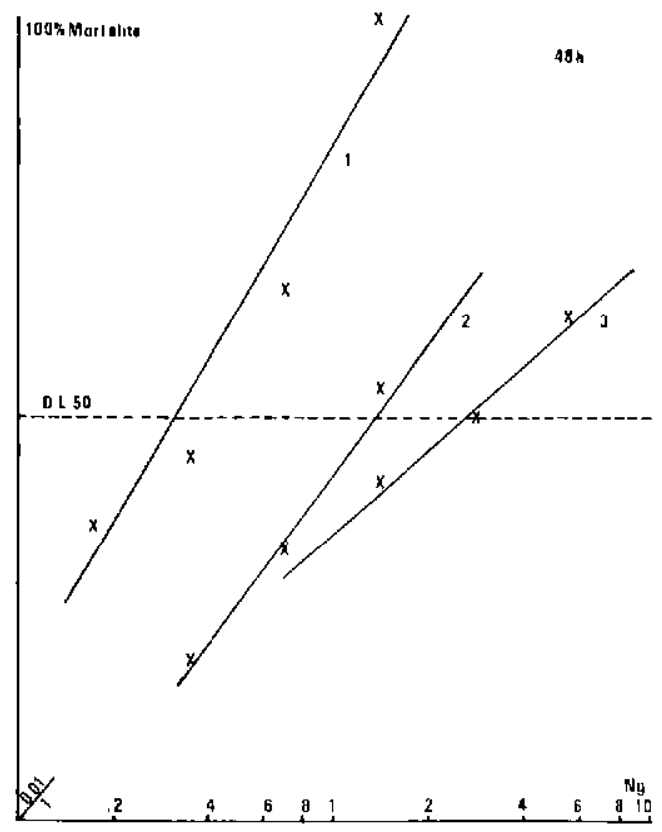

Graphique 2. -- Action de l'Endosulfan sur $G$, tachinoides (1), G. m. morsitans (2) et G. p. gambiensis (3).

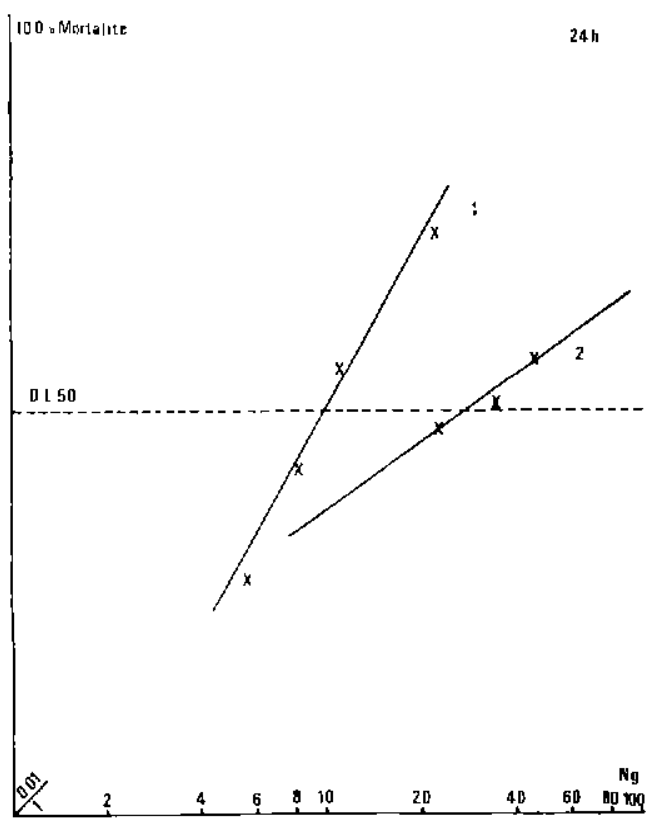

Graphique 3. - Action du D. D. T. sur $G$. tachinoides (1) et G. m. morsitans (2).

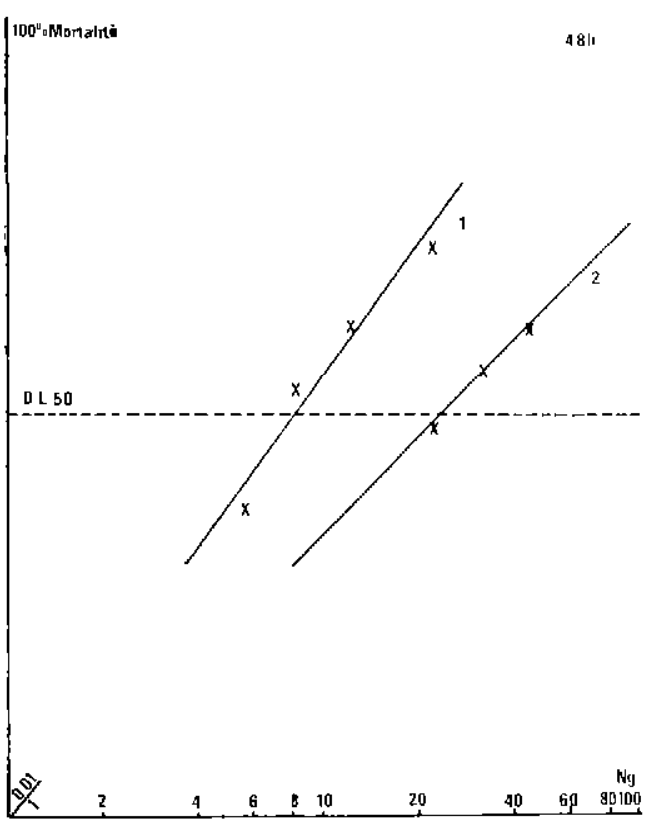

Graphique 4. - Action du D. D. T. sur G. tachinoides (1) et G. m. morsitans (2). 


\section{DISCUSSION}

Ces résultats concordent avec ceux obtenus par d'autres auteurs, notamment HADAWAY et ses collaborateurs $(2,4,3)$.

Bien qu'HADAWAY ait effectué les applications topiques des insecticides qu'il a expérimentés avec une microseringue $(0,025$ à $0,05 \mu l$ par mouche), et bien que le solvant utilisé ait été la diisobutylcétone, il est possible en effet de comparer les données fournies par cet auteur avec nos propres observations, compte tenu de ce que les D. L. 50 ont été, dans les 2 cas, calculées à partir des mortalités observées $48 \mathrm{~h}$ après la manipulation réalisée sur des individus ténéraux dans les $24 \mathrm{~h}$ suivant l'éclosion.

D. L. 50 obtenues en $\mathrm{ng} /$ mouche :

- Endosulfan :

avec G. morsitans: pour BREARD 1,35; pour HADAWAY 4,5 (coeff. $\times: 3,33$ ),

avec $G$. palpalis : pour BREARD 2,6; pour HADAWAY 7,7 (coeff. $\times 2,96$ ), avec $G$. tachinoides: BREARD 0,31.

- D. D. T. :

avec G. morsitans: pour BREARD 23,7; pour HADAWAY 72 (coeff. $\times: 3,04$ ),

avec $G$. tachinoides: BREARD 8,1.

Dans les 3 cas où les espèces sont identiques, les D. L. 50 obtenues par HADAWAY sont environ 3 fois plus élevées que celles que nous avons observées. Les différences de sensibilité entre espèces et insecticides sont remarquablement constantes.

\section{CONCLUSION}

De ces essais et de ceux précédemment effectués par d'autres auteurs, il se confirme que les diverses espèces de glossines étudiées ont une sensibilité différente aux insecticides organochlorés. Aucune explication n'a jusqu'ici été donnée sur les raisons possibles de ce phénomène. SAUVEL, se basant sur les observations de PRUDHAN qui avait constaté, en 1952, que les cristaux de D. D. T. se dissolvaient d'autant plus rapidement dans la cuticule d'insectes divers que le point de fusion de leurs cires étaient plus bas, a émis l'hypothèse, dans sa thèse de Doctorat Vétérinaire, que ces inégalités interspécifiques de sensibilité aux insecticides pourraient tenir à des différences de constitution des cires cuticulaires des diverses espèces de glossines.

Le matériel de l'O. M. S., dont l'utilisation ne présente aucune difficulté, donne des résultats très concordants avec ceux obtenus, au moyen de techniques plus élaborées, par d'autres auteurs.

Il nous paraît donc pouvoir convenir très bien pour évaluer, sur le terrain, la sensibilité des glossines aux insecticides, à condition d'opérer avec les solutions acétoniques ou avec un solvant comme la butanone (méthyl-éthylcétone) qui est moins volatil, sur des insectes ténéraux, et de prolonger les observations pendant une durée suffisante (au minimum $48 \mathrm{~h}$ ).

Au sujet de la composition des solvants utilisés, il est utile d'insister sur la haute toxicité du mélange D. O. P. + acétone par rapport à l'acétone seule.

\section{Remerciements}

Nous adressons tous nos remerciements à l'O. M. S., qui nous a procuré la trousse standard et les insecticides utilisés dans ce travail, au Dr J. ITARD, Chef du Service d'Entomologie de l'I. E. M. V. T., qui nous a fourni les insectes soumis aux épreuves et qui a relu et critiqué notre manuscrit, et à $M$. P. GUILLET, de I'O. R. S. T. O. M., qui nous a guidé lors de nos essais préliminaires.

\section{SUMMARY}

Evaluation of sensitivity to D. D. T. and Endosulfan of 3 species of laboratory bred glossinae using W.H. O. capillary applicators

The sensitivity of 3 species of laboratory bred glossinae $(G$, tachinoides, G. m. morsitans, G. p. gambiensis) to D. D. T. and Endosulfan, was tested by topical applications using W. H. O. capillary applicators.

This applicator handling is very simple and it gives results qualitatively comparable to those obtained by more sophisticated means.

The most sensitive glossina to Endosulfan is $G$. tachinoides. G. m. morsitans resistance is intermediate between that of $G$. tachinoides and that of $G . p$. gambiensis. 
Compared with Endosulfan, D. D. T. has a very low toxicity since the L. D. 50 obtained with this insecticide are about 20 times lower.

Among the 3 species of glossinae used, $G$, tachinoides is the most sensitive to both insecticides. $G$. m. morsitans mortality rate ranges between that of G. tachinoides and that of G.p. gambiensis.

\section{RESUMEN}

\section{Evaluación de la sensibilidad de 3 especies de glosinas de crianza para con el D. D. T. y el Endosulfan determinada por medio de aplicadores capilares de la $O$. M. S.}

Se probó la sensibilidad de 3 especies de glosinas de crianza ( $G$. tachinoides, G. m. morsitans, G. p. gambiensis) para con el D. D. T. y el Endosulfan por aplicaciones tópicas por medio del tubo capilar aplicador de la Organización mundial de la salud (O. M. S.).

Este, de un manejo muy sencillo, permite llegar a resultados comparables, cualitativamente, con los obtenidos por otros autores por medio de un material más sofisticado.

La especie de glosina más sensible al Endosulfan es $G$. tachinotdes; la resistencia de $G, m$. morsitans es intermediaria entre la de $G$, tachinoides y la de G. p. gambiensis.

En comparación con el Endosulfan, el D. D. T. presenta una toxicidad muy reducida ya que las D. L. 50 obtenidas con dicho insecticido son unas 20 vecez inferiores.

De las 3 especies de glosinas utılizadas, G. tachinoides es la más sensıble para con los 2 insecticidos. La mortalidad en $G$. m. morsitans es intermediaria entre $G$. tachinotdes y $G$. p. gambiensis.

\section{BIBLIOGRAPHIE}

1. CHALLIER (A.). Sensibilité de Glossina palpalis gambiensis Vanderplank, 1949 au D. D. T. et à la Dieldrine, déterminée au moyen de la trousse standard O. M. S. pour moustiques adultes. Bull. Sac. Path. exot., 1963,56 (3) : 519-533.

2. HADAWAY (A. B.). Toxicity of msecticides to tsetse flies. Bull. Org. mond. Santé, 1972, 46 (3) : 353362.

3. HADAWAY (A. B.). Insecticides for tsetse control. Joint W. H. O. Expert Committee and F. A. O. Expert Consultation on the African Trypanosomiasis, Rome, 8-12 November 1976.

4. HADAWAY (A. B.), TURNER (C. R.). Toxicity of insecticides to tsetse flies. Genève, O. M. S., 1975.

5. O. M. S. Méthode à suivre pour détermıner la sensibilité ou la résistance des moustiques adultes aux insecticides. $\mathrm{In}$ : 13e rapport du Comité O.M.S. d'experts des insecticides. Genève, O. M. S, 1963 , (série des rapports techniques, $\mathbf{n}^{\circ} 265$ ). Annexe $1: 45$ 53.

6. O. M. S. Méthode proposée pour déterminer la sen- sibılıté ou la résistance des mouches aux insecticides : mouches domestiques, glossines, stomoxes, calliphorines, etc. In: $17^{\mathrm{e}}$ rapport du Comité O. M.S. d'experts des insecticides. Genève, O. M. S., 1970, (série des rapports techniques: $\mathrm{n}^{0} 443$ ). Annexe 9: 126-131.

7. RIORDAN (K.). Measurements by two methods of the susceptibility to D. D. T. and dieldrin of Glossina palpalis (R.-D.) in Northern Nigeria. Bull. ent. Res., 1966, $57: 49-59$.

8. RIORDAN (K.). Further measurements, with the World Health Organization standard test kit, of the susceptibility to D. D.T. and dieldrin of Glossina palpalis R. D. in Nigeria. O. A. U./S. T. R. C., 12th meeting, Bangui, 1968, $102: 231-235$.

9. SAUVEL (Ch.). Glossines et insecticides. Thèse Doct. vét. Alfort, 1977, n 48.

10. SWAROOP (S.), GILROY (A. B.), VEMURA (K.), Méthodes statistiques utilisables dans les campagnes d'éradication du paludisme. Genève, O. M. S., 1968 , (série de monographies : $\mathrm{n}^{\circ} 5 \mathrm{l}$ ), $164 \mathrm{p}$. 